\title{
Improving Battery Performance via Mechanical Activation Enhanced Synthesis
}

\author{
L. Shaw, ${ }^{1}$ S.-J. Chiang, ${ }^{1}$ M. Luo, ${ }^{1}$ Z. Wang, ${ }^{1}$ M. Burrill, ${ }^{1}$ and A. Ortiz ${ }^{2}$ \\ ${ }^{1}$ Department of Mechanical, Materials and Aerospace Engineering, \\ Illinois Institute of Technology, Chicago, Illinois, USA \\ ${ }^{2}$ Departamento de Ingeniería Mecánica, Energética y de los Materiales, \\ Universidad de Extremadura, Badajoz, Spain
}

\begin{abstract}
Rechargeable batteries will play a critical role in vehicle electrification, utilization of renewable energy, and the construction of smart city and IoT (internet of things). For these emerging applications, rechargeable batteries with high energy density, fast charging capability, as well as low fabrication cost and long cycle life are urgently needed. This presentation focuses on synthesis of advanced materials to enhance performance of sodium ion batteries (SIBs) with liquid electrolytes or solid electrolytes for large scale, stationary energy storage where ultralong cycle life, high round trip efficiency, low cost, and high safety are important, while the high gravimetric energy densities offered by Li-ion batteries (LIBs) are not critical. To achieve long-cycle life and high safety, we have developed a mechanical-activation-enhanced reactions (MAER) method to synthesize Na-cathode material and $\mathrm{Na}$-ion conductor with controlled structural defects and larger $\mathrm{Na}$ diffusion pathways. Using this MAER method, we have achieved one of the best cycle stabilities of $\mathrm{O} 3-\mathrm{NaCrO}_{2}$ cathodes over 300 charge/discharge cycles without doping and one of the highest $\mathrm{Na}$ ion conductivities of $\mathrm{Na}_{3} \mathrm{Zr}_{2} \mathrm{Si}_{2} \mathrm{PO}_{12}$ solid electrolyte at room temperature $\left(>10^{-3} \mathrm{~S} / \mathrm{cm}\right)$. Detailed structural analyses reveal that MAER can minimize $\mathrm{Cr}^{3+}$ ion misplacement at $\mathrm{Na}$ sites to improve the cycle stability of $\mathrm{O} 3-\mathrm{NaCrO}_{2}$ and increase the bottleneck size of $\mathrm{Na}_{3} \mathrm{Zr}_{2} \mathrm{Si}_{2} \mathrm{PO}_{12}$ crystals to enhance its $\mathrm{Na}$ ion conductivity at room temperature. These studies have provided a new direction and offered guidelines to synthesize high performance Na-ion battery materials in the near future.
\end{abstract}

